\title{
Gravity cells as a necessary link in string theory. Formula and value of the gravitational constant in the region of a black hole. The frequency of gravitational waves. Formula and calculation of the electron mass.
}

Andrey Chernov ( $\sim$ and8591@gmail.com )

none Company/Institution

\section{Article}

Keywords: Black hole, gravitational cells, gravitational field, elementary charge, Schwarzschild radius formula, gravitational constant formula, gravitational cell mass, electron mass formula, electron mass calculation, string theory, gravitational strings, gravitational string vibration frequency, string vibration 3 frequency formula, gravitational waves, the formula for the frequency of gravitational waves

Posted Date: July 13th, 2021

DOl: https://doi.org/10.21203/rs.3.rs-622706/v3

License: (1) This work is licensed under a Creative Commons Attribution 4.0 International License. Read Full License 
Gravity cells as a necessary link in string theory. Formula and value of the gravitational constant in the region of a black hole. The frequency of gravitational waves. Formula and calculation of the electron mass.

\author{
Author Andrey Chernov \\ Address: Andrey Chernov, 16 Polenova str, apt.7, Irkutsk, 664007, Russia. \\ cell phone: +7 9645407 298. E mail: and8591@gmail.com
}

\title{
Content
}

1. Abstract -2 pg.

2. Introduction $-3-4 \mathrm{pg}$.

3. Methods $-4-15 \mathrm{pg}$.

4. Results and discussion $-15-16 \mathrm{pg}$.

5. Conclusions $-16-18 \mathrm{pg}$.

6. Declarations $-19 \mathrm{pg}$. 


\begin{abstract}
.
In this study, a new concept is introduced - gravitational cells. The body of a black hole consists of a huge number of such cells. Based on this hypothesis, using string theory, the Schwarzschild radius formula and the Coulomb formula, a formula for the gravitational constant was obtained. The new formula was used to determine the value of the gravitational constant in the region of black holes, and also confirmed the value of the usual gravitational constant. Also in this work, a new physical constant was obtained - the mass of the gravitational cell of a black hole. The results obtained made it possible to draw conclusions regarding the gravitational mass of the proton and the electron.
\end{abstract}

In this study, the gravity cell hypothesis was organically integrated into string theory. As a result, it was possible to apply the Planck formula to the gravitational interaction. Thus, the formula for the vibration frequency of gravitational strings (the frequency of gravitational waves) was obtained, and their frequency was determined. The formula for the mass of an electron was obtained. The electron mass calculated by the formula coincided with the experimental value.

Keywords. Black hole, gravitational cells, gravitational field, elementary charge, Schwarzschild radius formula, gravitational constant formula, gravitational cell mass, electron mass formula, electron mass calculation, string theory, gravitational strings, the quantum of the gravitational field, the formula for the quantum of the 
gravitational field, gravitational waves, the formula for the vibrations of gravitational strings.

\section{Introduction.}

Before moving on to the main part of the article, we briefly outline some of the main points that are understood in this study. Let's start with the formula for the gravitational constant.

In physics, there have been several attempts to propose scientifically substantiated formulas for calculating the gravitational constant. All the formulas presented by the authors are quite complex, and they necessarily contain additional (often artificial) coefficients and indicators, without which these formulas do not work. Also, in most of these formulas, as a rule, there is no elementary charge, but at the same time in all formulas there is mass in the form of an electron, nucleon, etc. (Looking ahead, we note that these are two main points that distinguish the new formula for the gravitational constant in the black hole region $\boldsymbol{G}_{\mathbf{0}}=\sqrt{\mathbf{2}} \boldsymbol{q}_{\mathbf{0}} \boldsymbol{c}$, where both of these factors are absent).

String theory is a branch of theoretical physics that studies the dynamics of the interaction of objects not as point particles, but as one-dimensional extended objects, the so-called quantum strings. String theory combines the ideas of quantum mechanics and the theory of relativity. The theory that arose to describe hadronic physics, but did not quite fit for this, turned out to be a kind of experiment in a vacuum. The main problem of string theory is expressed in a huge 
variety of possible solutions, the so-called "landscape problem". This significant circumstance, according to such famous scientists as Lee Smolin and David Gross, takes string theory out of the framework of scientific theory.

\section{Methods.}

The study begins with the Coulomb formula. Let's write this formula for the case of interaction of two opposite elementary charges:

$$
f=k \cdot \frac{q_{0} \cdot q_{0}}{r^{2}} \quad \text { or } \quad f=k \cdot \frac{q_{0}{ }^{2}}{r^{2}}
$$

Where $\boldsymbol{f}$ is the force of attraction of two elementary charges.

where $\boldsymbol{q}_{\mathbf{0}}$ is an elementary charge, $1,60217733 \cdot 10^{-19} \mathrm{C}$.

$\boldsymbol{k}$ is the coefficient of proportionality, $\boldsymbol{k}=\frac{\mathbf{1}}{\mathbf{4 \pi \varepsilon _ { 0 } \varepsilon}}$. Where $\frac{\mathbf{1}}{\mathbf{4 \pi \varepsilon _ { 0 }}}$ is a constant equal to $\mathbf{9} \cdot \mathbf{1 0}^{9} \frac{\mathrm{kg} \mathrm{m}}{\mathrm{C}^{2} \mathrm{~s}^{2}}$, and $\boldsymbol{\varepsilon}$ is the relative dielectric constant of the medium.

$\boldsymbol{r}$ is the distance between charges, $\mathrm{m}$.

The value of the proportionality coefficient $\boldsymbol{k}$ depends on the medium.

Maximum value $\boldsymbol{k}=\mathbf{9} \cdot \mathbf{1 0} 9 \frac{\mathrm{kg} \mathrm{m}}{\mathrm{C}^{2} \mathrm{~s}^{2}}$ in vacuum (where $\boldsymbol{\varepsilon}=1$ ), for alcohol $\boldsymbol{k}=2,6$. $10^{8}$ (where $\left.\boldsymbol{\varepsilon}=35\right)$, for water $\boldsymbol{k}=1,1 \cdot 10^{8}$ (where $\boldsymbol{\varepsilon}=80$ ), for copolymers $\boldsymbol{k} \geq$ $10^{4}$ (where $\boldsymbol{\varepsilon} \leq 105$ ), etc. Theoretically, $\boldsymbol{k}$ can approach its minimum value equal to $\mathbf{1}$. But $\boldsymbol{k}<\mathbf{1}$ cannot be in any medium, because then the fractionality of elementary charges is allowed. Practical confirmation of this lies in the fact that science does not know substances where $\boldsymbol{\varepsilon}>\boldsymbol{9} \cdot \mathbf{1 0}^{\mathbf{9}}$. The maximum value of $\boldsymbol{\varepsilon}$ is recorded for metal nanoisland structures, $10^{7}-10^{8}$, where $\boldsymbol{k} \geq 90$. 
Now let's move on to the gravitational interaction of such physical objects as black holes. (The result obtained here will then make it possible to pass to the gravitational interaction of ordinary bodies).

So, we have two black holes of mass $\boldsymbol{M}$ and $\boldsymbol{M}_{\mathbf{1}}$, located at a distance $\mathrm{r}$ from each other. The body of a black hole consists of many superdense cells with mass $\boldsymbol{m}_{\mathbf{0}}$ and charge $\boldsymbol{q}_{\boldsymbol{g}}$, numerically equal to the value of two elementary charges $\boldsymbol{q}_{\mathbf{0}}$, that is, $\boldsymbol{q}_{\boldsymbol{g}}=\mathbf{2} \boldsymbol{q}_{\mathbf{0}}$. Such a cell (let's call it a gravitational cell), in a black hole, is a tightly compressed pair of elementary particles: an electron and a proton. In this case, the mass of such a cell will be less than the total mass of a free proton and a free electron by $\Delta \boldsymbol{m}$ due to the release of energy during compression, where $\Delta \boldsymbol{m}=\boldsymbol{E} / \mathrm{c}^{2}$. The gravitational cells of one black hole $\boldsymbol{M}$ are connected by ultramicroscopic strings with the gravitational cells of another black hole $\boldsymbol{M}_{\mathbf{1}}$. As a result, a common gravitational field $\boldsymbol{E}$ is created in space, through which the interaction between black holes takes place along the gravitational strings. The field strength of the gravitational cell $\boldsymbol{g}_{\mathbf{0}}$ in the black hole region $\boldsymbol{M}$ is determined by the following formula:

$$
g_{0}=\frac{k_{0} q_{g 1} \cdot k_{0} q_{g 2}}{r^{2}} \quad \text { or } \quad g_{0}=k_{0}^{2} \frac{4 q_{0}^{2}}{r^{2}}
$$

where $\boldsymbol{g}_{\mathbf{0}}$ is the value of the gravitational field of one gravitational cell of a black hole, $\mathrm{m} / \mathrm{s}^{2}$.

$\boldsymbol{k}_{\mathbf{0}}-$ proportionality coefficient, $\boldsymbol{k}_{\mathbf{0}}=\mathbf{1} \frac{\mathrm{m}^{3 / 2}}{\mathrm{C} \mathrm{s}}$

$\boldsymbol{q}_{\mathbf{0}}$ - elementary charge $1,60217733 \cdot 10^{-19} \mathrm{C}$. 
$\boldsymbol{q}_{\boldsymbol{g} \mathbf{1}}$ and $\boldsymbol{q}_{\boldsymbol{g} \mathbf{2}}$ are the charges of the gravitational cells of black holes, $\boldsymbol{q}_{\boldsymbol{g}}=\mathbf{2} \boldsymbol{q}_{\mathbf{0}}=$ $3,20435466 \cdot 10^{-19} \mathrm{C}$.

$\boldsymbol{r}$ - distance between cells, $\mathrm{m}$.

Thus, the gravitational field around the gravitational cell at a distance $\boldsymbol{r}=\mathbf{1}$

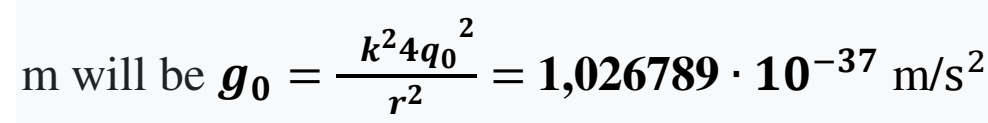

Formula (1-2) is similar to Formula (1-1). But if in the Coulomb formula the coefficient of proportionality in vacuum is $\boldsymbol{k}=\mathbf{9} \cdot \mathbf{1 0}^{\mathbf{9}}$, then in the formula (1-2) $\boldsymbol{k}_{\mathbf{0}}=\mathbf{1}$. The reason for such a large discrepancy in the coefficients is that the substance of the black hole is so strongly compressed that almost all lines of force of elementary charges are closed inside gravitational cells. And only an extremely small part of the lines of force go out from the cell, creating a gravitational field in the outer space. As a result of this circumstance, the coefficient of proportionality of elementary charges outside the gravitational cell decreases to its minimum threshold, that is, exactly to 1 . At the same time, the main electric field, with the coefficient of proportionality $\boldsymbol{k}=\mathbf{9} \cdot \mathbf{1 0}^{\mathbf{9}}$, remains closed between elementary charges inside the gravitational cells and therefore does not manifest itself in any way.

Now let's consider the interaction of two black holes $\boldsymbol{M}$ and $\boldsymbol{M}_{\mathbf{1}}$. The body of a black hole with mass $\boldsymbol{M}$ consists of a huge number of gravitational cells with mass $\boldsymbol{m}_{\mathbf{0}}$. Therefore, the total number of such cells will be: $\boldsymbol{n}=\frac{\boldsymbol{M}}{\boldsymbol{m}_{\mathbf{0}}}$. These gravitational cells form a common gravitational field in space, equal to $\boldsymbol{E}=\boldsymbol{g}_{\mathbf{0}}$. 
$\boldsymbol{n}$. Thus, the gravitational field $\boldsymbol{E}$ around a black hole $\boldsymbol{M}$ when it interacts at a distance $\mathrm{r}$ with another black hole will be:

$$
E=\frac{k_{0}^{2} 4 q_{0}^{2}}{r^{2}} \frac{M}{m_{0}} \quad \text { or } \quad E=\frac{k_{0}^{2} 4 q_{0}^{2}}{m_{0}} \frac{M}{r^{2}}
$$

The expression $\frac{\boldsymbol{k}_{0}^{2} \mathbf{4 \boldsymbol { q } _ { 0 }}}{\boldsymbol{m}_{\mathbf{0}}}$ is the value of the gravitational constant $\boldsymbol{G}_{\mathbf{0}}$ for the case of interaction of two black holes. As a result, formula (1-3) will take the following form:

$$
E=G_{0} \frac{M}{r^{2}}, \text { where } G_{0}=\frac{k_{0}^{2} 4 q_{0}^{2}}{m_{0}}
$$

It follows that $\boldsymbol{m}_{0}=\frac{\boldsymbol{k}_{0}^{2} \mathbf{4 q _ { 0 }}}{G_{0}}$. (Further, for a better perception of information, the coefficient $\boldsymbol{k}_{\mathbf{0}}$ will not be displayed in the formulas, due to the fact that $\boldsymbol{k}_{\mathbf{0}}=$ 1). It is possible to determine the value $\boldsymbol{m}_{\mathbf{0}}$ if we admit the possibility of equality $G_{0}=G=6,6743 \cdot 10^{-11}$. In this case, we get $m_{0}=\frac{4 q_{0}^{2}}{6,6743 \cdot 10^{-11}}=1,53842$. $10^{-27} \mathrm{~kg}$. But such a calculation of the value $\boldsymbol{m}_{0}$ cannot be considered correct, because the value of the gravitational constant $G_{0}$ in the region of the black hole may have a different value, that is, $\boldsymbol{G}_{\mathbf{0}} \neq \boldsymbol{G}$. Therefore, in order to correctly determine the value of $\boldsymbol{m}_{\mathbf{0}}$ and also the value of $\boldsymbol{G}_{\mathbf{0}}$, it is necessary to turn to the formula for the Schwarzschild gravitational radius:

$$
R=\frac{2 G_{0}}{c^{2}} \cdot M
$$

where $\boldsymbol{R}$ is the gravitational radius of a black hole, $\boldsymbol{G}_{\mathbf{0}}$ is the gravitational constant in the field of a black hole, $\boldsymbol{M}$ is the mass of a black hole, and $\boldsymbol{c}$ is the speed of light. 
In this formula, of particular interest is the expression $\frac{2 G_{0}}{c^{2}}$, which is measured in "m / kg", and denotes the specific length of the gravitational radius of a black hole. (In what follows, we denote $\frac{2 G_{0}}{\boldsymbol{c}^{2}}$ as $\boldsymbol{\gamma}$ ). And now a very important point. In space compressed to a point around a black hole, such a category as space does not exist. Therefore, in the Schwarzschild radius, all physical quantities merge into one quantity - mass, where the initial dimensional unit is the mass of the gravitational cell $\boldsymbol{m}_{\mathbf{0}}$. As a result of this circumstance, in the gravitational radius of the black hole, the numerical equality must be observed: $\gamma=m_{0}$. Taking into account that $\gamma=\frac{2 G_{0}}{c^{2}}$, and $m_{0}=\frac{4 q_{0}^{2}}{G_{0}}$, we get the following equation: $\frac{2 G_{0}}{c^{2}}=\frac{4 q_{0}{ }^{2}}{G_{0}}$. Let's solve it and as a result we get:

$$
\begin{aligned}
& G_{0}=\sqrt{2} q_{0} c=6,7927 \cdot 10^{-11} \frac{\mathrm{m}^{3}}{\mathrm{~s}^{2} \mathrm{~kg}} \\
& m_{0}=\frac{\sqrt{8} q_{0}}{c}=1,511593 \cdot 10^{-27} \mathrm{~kg}
\end{aligned}
$$

As you can see, $\boldsymbol{G}_{\mathbf{0}}$ differs from $\boldsymbol{G}=\mathbf{6 , 6 7 4 3} \cdot \mathbf{1 0}^{\mathbf{- 1 1}}$ by $1,7 \%$. In this case, the value of $\boldsymbol{G}_{\mathbf{0}}$ is related to the mass of the gravitational cell $\boldsymbol{m}_{\mathbf{0}}$ by the following beautiful formula: $G_{0}=\frac{m_{0} c^{2}}{2}(\mathbf{1 - 9})$.

Now consider the gravitational interaction of an ordinary (that is, not superdense) body $\boldsymbol{M}$ with another body $\boldsymbol{M}_{\mathbf{1}}$. The formula of the gravitational field around a body of mass $\boldsymbol{M}$ in this case will look like this:

$$
E=\frac{k^{2} 4 q_{0}^{2}}{r^{2}} \frac{M}{m}=\frac{k^{2} 4 q_{0}^{2}}{m} \frac{M}{r^{2}} \quad \text { or } \quad E=G \frac{M}{r^{2}}
$$


Where $\boldsymbol{k}$ is the proportionality coefficient of the charge of the gravitational cell of an ordinary body, where $\boldsymbol{k}=\mathbf{1}, \mathbf{0 3 8 9 3 1} \frac{\mathrm{m}^{3 / 2}}{\mathrm{C} \mathrm{s}}, \boldsymbol{k}^{2}=\mathbf{1 , 0 7 9} 378 \frac{\mathrm{m}^{3}}{\mathrm{C}^{2} \mathrm{~s}^{2}}$ $\boldsymbol{m}$ is the mass of the gravitational cell, where $\boldsymbol{m}=\mathbf{1 , 6 6 0 5 3 9} \cdot \mathbf{1 0}^{-\mathbf{2 7}} \mathrm{kg}$ $\boldsymbol{G}$ - gravitational constant, where $\boldsymbol{G}=\mathbf{6 , 6 7 4 3} \cdot \mathbf{1 0}-\mathbf{1 1} \frac{\mathrm{m}^{3}}{\mathrm{~s}^{2} \mathrm{~kg}}$ The gravitational field around the gravitational cell at a distance of $\boldsymbol{r}=\mathbf{1} \mathrm{m}$ will be $g=\boldsymbol{k}_{0}^{2} 4{q_{0}}^{2}=1,108293 \cdot 10^{-37} \mathrm{~m} / \mathrm{s}^{2}$

Now let's explain the magnitude of $\boldsymbol{k}$ and $\boldsymbol{m}$. Let's start with $\boldsymbol{m}=$ $\mathbf{1 , 6 6 0 5 3 9} \cdot \mathbf{1 0}^{-\mathbf{2 7}} \mathrm{kg}$. To do this, imagine that two bodies of mass $\boldsymbol{M}$ and $\boldsymbol{M}_{\mathbf{1}}$ were formed from two black holes $\boldsymbol{M}^{\prime}$ and $\boldsymbol{M}^{\prime}{ }_{\mathbf{1}}$. In this case, each gravitational cell of the black hole, due to the arrival of energy $\boldsymbol{E}$, increases its mass $\boldsymbol{m}_{\mathbf{0}}$ to mass $\boldsymbol{m}$ by the amount $\Delta \boldsymbol{m}$ (where $\Delta \boldsymbol{m}=\boldsymbol{E} / \mathrm{c}^{2}$ ). As a result, a plasma is formed from the superdense matter of a black hole, from which gaseous, liquid and solid substances can be formed. All four states of matter are neutral, that is, they have a total electric charge equal to zero. As a result of this circumstance, any substance can be represented as a huge set of gravitational cells. These cells consist of a proton and an electron with a total charge of $\mathbf{2} \boldsymbol{q}_{\mathbf{0}}$, as well as of neutrons, which are also a pair of a proton and an electron with a total charge of $\mathbf{2} \boldsymbol{q}_{\mathbf{0}}$. Thus, the mass of the gravitational cell $\boldsymbol{m}$ of any non-superdense substance (plasma, gas, liquid and solid) with high accuracy will be equal to $\mathbf{1} \mathbf{D a}$ (this is $1 / 12$ of the mass of an atom of the isotope of carbon-12) or $\mathbf{1 , 6 6 0 5 3 9} \cdot \mathbf{1 0}^{-\mathbf{2 7}} \mathrm{kg}$. This implies that:

$$
k=\sqrt{\frac{G m}{4 q_{0}{ }^{2}}}=\sqrt{\frac{6,6743 \cdot 10^{-11} m}{4 q_{0}^{2}}}=1,038931
$$


Thus, $\boldsymbol{k}>\boldsymbol{k}_{\mathbf{0}}$. This can be explained by the fact that, in contrast to the gravitational cell of a black hole, where everything is tightly compressed, in an ordinary cell there is an ultramicroscopic distance between elementary charges. As a result of this circumstance, more lines of force come out of an ordinary cell and go out, creating a 7,9\% stronger gravitational field in outer space. But due to the fact that the mass of an ordinary gravitational cell $\boldsymbol{m}$ is more than the mass of the gravitational cell of a black hole $\boldsymbol{m}_{\mathbf{0}}$ by $\mathbf{9 . 9 \%}$, then $1 \mathrm{~kg}$ of ordinary matter has a smaller number of gravitational cells than $1 \mathrm{~kg}$ of black hole matter. As a result of this circumstance, it turns out that $\boldsymbol{G}<\boldsymbol{G}_{\mathbf{0}}$, even though $\boldsymbol{k}>\boldsymbol{k}_{\mathbf{0}}$. For clarity, we will show this in a mathematical form:

$$
G=\frac{k^{2} 4 q_{0}{ }^{2}}{m}=\frac{1,079378 \cdot 4 q_{0}{ }^{2}}{1,660539 \cdot 10^{-27}}<G_{0}=\frac{k_{0}^{2} 4 q_{0}^{2}}{m_{0}}=\frac{4 q_{0}^{2}}{1,511593 \cdot 10^{-27}}
$$

Next, consider the gravitational interaction of a black hole with mass $\boldsymbol{M}_{\mathbf{0}}$ and an ordinary body with mass $\boldsymbol{M}$ at a distance $\boldsymbol{r}$ between them.

1. The gravitational field $\boldsymbol{E}$ around a black hole with mass $\boldsymbol{M}_{\mathbf{0}}$ will be:

$$
E=\frac{k_{0} k 4 q_{0}^{2}}{r^{2}} \frac{M_{0}}{m_{0}}=\frac{k_{0} k 4 q_{0}^{2}}{m_{0}} \frac{M_{0}}{r^{2}} \quad(1-13) \text { or } E=G_{1} \frac{M_{0}}{r^{2}}
$$

where $\boldsymbol{k}_{\mathbf{0}}=\mathbf{1}, \boldsymbol{k}=\mathbf{1}, \mathbf{0 3 8 9 3 1}, \boldsymbol{m}_{\mathbf{0}}=\mathbf{1}, \mathbf{5 1 1 5 9 3} \cdot \mathbf{1 0}^{-\mathbf{2 7}} \mathrm{kg}$. Hence the gravitational constant is equal to:

$$
G_{1}=\frac{k_{0} k 4 q_{0}{ }^{2}}{m_{0}}=\frac{1,038931 \cdot 4 q_{0}^{2}}{1,511593 \cdot 10^{-27}}=7,0572 \cdot 10^{-11} \frac{\mathrm{m}^{3}}{\mathrm{~s}^{2} \mathrm{~kg}}
$$

The gravitational field $\mathrm{E}$ around a black hole of mass $\mathrm{M}$ will be:

$$
E=\frac{k_{0} k 4 q_{0}^{2}}{r^{2}} \frac{M}{m}=\frac{k_{0} k 4 q_{0}^{2}}{m} \frac{M}{r^{2}}(1-14) \text { or } E=G_{2} \frac{M}{r^{2}}
$$


where $\boldsymbol{k}_{\mathbf{0}}=\mathbf{1}, \boldsymbol{k}=\mathbf{1}, \mathbf{0 3 8 9 3 1}, \boldsymbol{m}=\mathbf{1}, \mathbf{6 6 0 5 3 9} \cdot \mathbf{1 0}^{-27} \mathrm{~kg}$. Hence the gravitational constant is equal to:

$$
G_{2}=\frac{k_{0} k 4 q_{0}^{2}}{m}=\frac{1,038931 \cdot 4 q_{0}^{2}}{1,660539 \cdot 10^{-27}}=6,4242 \cdot 10^{-11} \frac{\mathrm{m}^{3}}{\mathrm{~s}^{2} \mathrm{~kg}}
$$

Now we apply the gravity cell hypothesis to string theory. Consider first the gravitational interaction between black holes, and then move on to the gravitational interaction between ordinary masses.

The gravitational field between black holes $\boldsymbol{M}_{\mathbf{0 1}}$ and $\boldsymbol{M}_{\mathbf{0 2}}$ consists of many ultramicroscopic vibrating strings that connect the gravitational cells of black holes. These gravitational strings in their totality form a gravitational field in space. From the set of energies e of these strings, the total energy of the gravitational field between the bodies is added, that is, $\boldsymbol{E}=\sum \boldsymbol{e}$. The vibration energy of one gravitational string e connecting two gravitational cells is a constant value and is numerically equal to $g_{0}(\mathbf{f} .1-3)$, that is, $\boldsymbol{e}=\mathbf{4} \boldsymbol{q}_{\mathbf{0}}{ }^{2}=\mathbf{1 , 0 2 6 7 8 9}$. $10^{-37} \mathrm{~J}$

Essentially, $\boldsymbol{e}$ is the energy quantum of the gravitational field. But the gravitational quantum of energy, in contrast to the electromagnetic quantum of energy (photon), does not move in space, but vibrates in the form of a long energy string connecting two gravitational cells of two masses. The energy source of one string is 4 elementary charges located in two gravitational cells. These charges vibrate with the same frequency $\gamma$ together with the gravitational string, forming a single whole with the string. (It should be noted that the frequency 


\section{of vibrations of gravitational strings can be considered as the frequency of}

\section{gravitational waves, because these are two names for one physical process).}

With a decrease (increase) in the distance $\mathrm{R}$ between the cells, an increase (decrease) in the frequency $\gamma$ of oscillations of the gravitational string occurs, but the energy of the quantum always remains unchanged: $\boldsymbol{e}=\mathbf{1}, \mathbf{0 2 6 7 8 9} \cdot \mathbf{1 0}^{-\mathbf{3 7}} \mathrm{J}$.

(This can be compared with playing on guitar when clamping the neck of the guitar changes the length of the string, after which the frequency of vibration of the string changes). In contrast to the well-known formula for the quantum of the electromagnetic field $\boldsymbol{e}=\boldsymbol{h} \boldsymbol{\gamma}$, the formula for the quantum of the gravitational field is as follows:

$$
e=h_{g} \gamma R^{2} \quad(1-15) \quad \text { or } \quad \gamma=\frac{e}{h_{g} R^{2}}
$$

$\boldsymbol{e}$ is the energy of the quantum of the gravitational field, $\boldsymbol{e}=\mathbf{1 , 0 2 6 7 8 9} \cdot \mathbf{1 0}^{-\mathbf{3 7}} \mathrm{J}$. $\boldsymbol{h}_{\boldsymbol{g}}$ is Planck's constant for the case of gravitational interaction, where $\boldsymbol{h}_{\boldsymbol{g}}=\frac{\mathbf{1}}{\mathbf{4}} \boldsymbol{h}=$ $1,656518 \cdot 10^{-34}$ $\boldsymbol{\gamma}$ is the vibration frequency of the gravitational string, $c^{-1}$. $\boldsymbol{R}$ is the distance between cells (bodies), $\mathrm{m}$.

The change in the value of Planck's constant under conditions of a gravitational field is explained by the following reason. Unlike the electromagnetic field, where a quantum of energy (photon) is formed as a result of oscillations of one charged particle (electron), the quantum of the gravitational field is the result of oscillations of already 4 charges located in two opposite cells. It is this 
circumstance that is the reason for the 4 times decrease in the value of the Planck constant.

These charges are in each gravitational cell in the form of 2 particles: an electron $\boldsymbol{m}_{\boldsymbol{e}}$ and a positron $+\boldsymbol{m}_{\boldsymbol{e}}$. In this case, the rest of the mass of the gravitational cell has a neutral charge. Each meter of increase in the distance between bodies (cells) reduces the frequency of vibrations of the gravitational string by $\frac{\mathbf{1}}{\boldsymbol{R}^{2}}$ times. As a result, the frequency of vibrations of the string decreases from the maximum value of vibrations $\gamma_{\max }$ to an infinitely small value. Moreover, $\boldsymbol{\gamma}_{\max }$ is equal to:

$$
\gamma_{\max }=\frac{e}{h_{g}}=\frac{1,026789 \cdot 10^{-37}}{1,656518 \cdot 10^{-34}}=6,1984796 \cdot 10^{-4} c^{-1}
$$

Accordingly, along with a change in the vibrations of the string, the frequency of the vibrations of the charges in the cells changes.

And now an important point. The maximum oscillation frequency of an electron (positron) $\gamma_{\max }$ depends on the specific mass of an electron in the gravitational cell. That is, the maximum frequency $\gamma_{\max }$ is equal to the ratio of the electron mass $m_{e}$ to the total mass of the gravitational cell $m_{0}$, that is, $\gamma_{\max }=\frac{m_{e}}{m_{0}-m_{e}}(1-18)$

Let's check the correctness of this statement. To do this, we transform this formula (1-18) and then substitute the values $\boldsymbol{m}_{\mathbf{0}}$ and $\boldsymbol{\gamma}_{\boldsymbol{m a x}}$ into it. As a result, we get the value of the mass of the electron (positron):

$$
m_{e}=\frac{m_{0} \gamma_{\max }}{1+\gamma_{\max }}=\frac{1,511593 \cdot 10^{-27} \cdot 6,1984796 \cdot 10^{-4}}{1+6,1984796 \cdot 10^{-4}}=9,363775 \cdot 10^{-31} \mathrm{~kg}
$$


As you can see, the electron mass calculated by the formula almost completely coincided with the experimental mass of a free electron: $\mathbf{9 , 1 0 9 3 8 9}$ $\cdot \mathbf{1 0}^{-\mathbf{3 1}} \mathrm{kg}$. The reason for the discrepancy by $\mathbf{2 , 7 \%}$ may be the fact that in the gravitational cell of a black hole there is an insignificant change in the mass of an electron in comparison with the mass of an electron in a free state.

Further, taking into account that, $\boldsymbol{m}_{\mathbf{0}}=\frac{\sqrt{\mathbf{8}} \boldsymbol{q}_{\mathbf{0}}}{c}, \boldsymbol{\gamma}_{\max }=\frac{\boldsymbol{e}}{\boldsymbol{h}_{g}}, \boldsymbol{h}_{g}=\frac{\mathbf{1}}{\mathbf{4}} \boldsymbol{h}, \boldsymbol{e}=\mathbf{4} \boldsymbol{q}_{0}{ }^{2}$, we obtain the basic formula for the electron mass:

$$
m_{e}=\frac{32 \sqrt{2} q_{0}{ }^{3}}{\operatorname{ch}\left(1+\frac{16 q_{0}{ }^{2}}{h}\right)}
$$

\section{Note that on the right side of the formula there are independent} physical constants $q_{0}, h, c$, where the category "mass" is absent.

In the case of gravitational interaction between ordinary bodies, the energy of the gravitational string has a different value and is equal to $\boldsymbol{e}=\mathbf{1 , 1 0 8 2 9 3}$. $\mathbf{1 0}^{-37} \mathrm{~J}$ (1-11). As a result, we obtain, according to the formula (1-17), the maximum frequency of string vibrations:

$$
\gamma_{\text {max }}=\frac{e}{h_{g}}=\frac{1,108293 \cdot 10^{-37}}{1,656518 \cdot 10^{-34}}=6,69050 \cdot 10^{-4} c^{-1}
$$

As you can see, the mass of an electron in a "normal" gravitational cell is $18 \%$ than the mass of an electron in a superdense cell. This is explained by the increase in the mass of the gravitational cell by $10 \%$ and the related structural changes within the "normal" gravitational cell. Thus, the mass of an electron in a superdense gravitational cell is closest to the mass of an electron in a free state: the difference is only $2,7 \%$. 
(For better perception, in formulas 1-15, 1-16, 1-17, 1-20, conversion factors for units of measurement are not specially set, which are equal to 1).

\section{Results and discussion.}

In this study, gravitational constants were determined for different cases of gravitational interaction.

1. $\boldsymbol{G}_{\mathbf{0}}=\mathbf{6}, \mathbf{7 9 2 7} \cdot \mathbf{1 0}^{-\mathbf{1 1}}$ (for the case of interaction of two black holes).

2. $\boldsymbol{G}=\mathbf{6}, \mathbf{6 7 4 3} \cdot \mathbf{1 0}^{-\mathbf{1 1}}$ (for the case of interaction of two ordinary bodies).

3. $\boldsymbol{G}_{1}=\mathbf{7}, \mathbf{0 5 7 2} \cdot \mathbf{1 0}^{-11}$ and $\boldsymbol{G}_{2}=\mathbf{6}, \mathbf{4 2 4 2} \cdot \mathbf{1 0}^{-11}$ (for the case of interaction between a black hole and an ordinary body).

It is necessary to explain the third case of gravitational interaction, where there are two values of the gravitational constants $\boldsymbol{G}_{\mathbf{1}}$ and $\boldsymbol{G}_{\mathbf{2}}$. The presence of two gravitational constants can raise the question: "Isn't there a violation of Newton's third law?" There is no violation here, because throughout the study, mass was only a measure of the amount of matter, and nowhere did it act as a gravitational and inertial mass. This is clearly seen from formulas (1-13) and (114), in which the masses $\boldsymbol{M}_{\mathbf{0}}$ and $\boldsymbol{M}$ show only the number of gravitational cells in the body: $\boldsymbol{n}=\frac{\boldsymbol{M}_{\mathbf{0}}}{\boldsymbol{m}_{\mathbf{0}}}$ and $\boldsymbol{n}=\frac{\boldsymbol{M}}{\boldsymbol{m}}$. Therefore, Newton's third law $\left(\boldsymbol{F}_{\mathbf{1}}=-\boldsymbol{F}_{\mathbf{2}}\right)$, where there is inertial mass $\left(\boldsymbol{m}_{\mathbf{1}} \boldsymbol{a}_{\mathbf{1}}=-\boldsymbol{m}_{\mathbf{2}} \boldsymbol{a}_{\mathbf{2}}\right)$, cannot be applied here. Therefore, there is no contradiction in the fact that in one gravitational interaction there are two gravitational constants. 
When discussing the research results, it is necessary to pay attention to the following point. The force of interaction between two elementary charges $\boldsymbol{q}_{\mathbf{0}}$ inside the gravitational cell is relatively large and is determined by the formula (11), where the proportionality coefficient is $\boldsymbol{k}=\mathbf{9} \cdot \mathbf{1 0}^{\mathbf{9}}$. But outside the gravitational cell, the proportionality coefficient $\boldsymbol{k}$ decreases sharply and for superdense gravitational cells it becomes equal to 1 , for ordinary gravitational cells $-\mathbf{1 , 0 3 8 9 3 1}$. This circumstance $9 \cdot \mathbf{1 0}^{9}$ times weakens the electric field outside the gravitational cell, turning it into a gravitational field.

The scientific result of this study is the determination of the mass of the gravitational cell of a black hole $\boldsymbol{m}_{\mathbf{0}}=\mathbf{1}, \mathbf{5 1 1 5 9 3} \cdot \mathbf{1 0}^{\mathbf{- 2 7}} \mathrm{kg}$. Gravitational cells with a mass less than $\boldsymbol{m}_{\mathbf{0}}$ do not exist.

Obtaining the formula for the quantum of the gravitational field and the formula for the electron mass is a significant result of the research. The coincidence of the obtained electron mass with its experimental mass indicates the correctness of the new formula.

When discussing the results, it should be emphasized that the formula for the gravitational constant and the formula for the electron mass were obtained by combining the hypothesis of gravitational cells and string theory.

\section{Conclusions.}

An important result of this research is the addition of the gravitational cell hypothesis to string theory and the subsequent application of the enriched theory to 
one of the fundamental interactions - gravitational interaction. This addition avoided the main problem of string theory, which is expressed in a huge variety of possible solutions, the so-called "landscape problem". On this theoretical basis, it was possible to apply Planck's formula to the gravitational interaction of bodies. As a result, the formula for the quantum of the gravitational field and the formula for the vibration frequency of gravitational strings were obtained, and their maximum frequency was determined. Then, on this basis, the formula for the electron mass was obtained. The electron mass calculated by this formula almost completely coincided with the experimental value of the electron.

Through the application of the hypothesis of gravitational cells, the formula for the gravitational constant $\boldsymbol{G}_{\mathbf{0}}=\sqrt{\mathbf{2}} \boldsymbol{q}_{\mathbf{0}} \boldsymbol{c}$ was obtained and its value in the region of black holes was determined $\boldsymbol{G}_{\mathbf{0}}=\mathbf{6 , 7 9 2 7} \cdot \mathbf{1 0}^{-\mathbf{1 1}}$. Also received justification and confirmation of the value of the "usual" gravitational constant $G=6,6743 \cdot 10^{-11}$.

Thus, there are two specific results of combining the hypothesis of gravitational cells and string theory: these are the electron mass calculated by the formulas and the value of the gravitational constant, which coincided with the experimental data. Duplicate coincidence of results on the same theoretical basis cannot be accidental.

In this study, the mass of the gravitational cell of a black hole was determined, where $\boldsymbol{m}_{\mathbf{0}}=\mathbf{1}, \mathbf{5 1 1 5 9 3} \cdot \mathbf{1 0}^{-\mathbf{2 7}} \mathrm{kg}$. The value of the gravitational constant in the region of interaction of black holes depends on the value $\boldsymbol{m}_{\mathbf{0}}: \boldsymbol{G}_{\mathbf{0}}=$ 
$\frac{\boldsymbol{m}_{0} c^{2}}{2}$. In this case, gravitational cells with a mass less than $\boldsymbol{m}_{\mathbf{0}}$ do not exist. As a result, $\boldsymbol{m}_{\mathbf{0}}$ should be classified as new physical constants.

A detailed interpretation of the results obtained in this study will be given in the next article. Now at this stage, it is important to consolidate the results obtained. 


\section{Declarations}

1. Availability of data and materials.

All data obtained and analyzed in the course of this study is included in this article.

2. Competing interests. Not applicable (there are no competing interests).

3. Funding. Not applicable.

4. Authors' contributions. Not applicable.

5. Acknowledgements. Not applicable. 